\title{
MATLAB Simulation of IEEE802.11p Technology on High User's Mobility
}

abderrahim mountaciri ( $\nabla$ yahyamountaciri@gmail.com )

Université Hassan 1er de Settat: Universite Hassan 1er de Settat https://orcid.org/0000-0001-7604$290 X$

\section{Research Article}

Keywords: Mobility, phenomena, Doppler, Orthogonal Frequency Division Multiplexing

Posted Date: June 28th, 2021

DOI: https://doi.org/10.21203/rs.3.rs-540883/v1

License: (c) (i) This work is licensed under a Creative Commons Attribution 4.0 International License. Read Full License 


\title{
MATLAB Simulation of IEEE802.11p Technology on High User's Mobility
}

\author{
MY Aberrahim Mountaciri \\ Laboratoire d'Ingénierie, de Management Industriel et d'Innovation (LIMII), Faculté des Sciences et \\ techniques (FST), Hassan First Université of Settat, Settat, Morocco
}

\begin{abstract}
In this article proposed IEEE 802.11p Physical layer (PHY). A $M A T L A B$ simulation is performed to analyze the baseband processing of the transceiver. Orthogonal Frequency Division Multiplexing (OFDM) is applied in this project according to the IEEE 802.11 standard, which allows data transmission rates from 3 to 27 Mbps. Separate modulation schemes, bit phase shift modulation (BPSK), quadrate phase shift modulation (QPSK), and quadrature amplitude modulation (QAM), are used for different data rates. These schemes are combined with time interleaving and a convolutional error correction code. A guard interval is inserted at the start of the transmitted symbol to reduce the effect of intersymbol interference (ISI). This article studies the PHY physical layer of the IEEE 802.11p vehicular communication standard. An IEEE.802.11p PHY model, with many associated phenomena, is implemented in the V2V vehicle-tovehicle, and the vehicle-to-vehicle ad hoc network (VANET) provides convenient coordination between moving vehicles. A moving vehicle could move at a very high speed, producing a Doppler effect that damages OFDM symbols and also causes inter-carrier interference (ICI). This article has discussed VANET technology versus 802.11a technology, as they have many differences when it comes to user mobility. The Doppler effect resulting from the mobility of the user with a high speed of 25 to $400 \mathrm{~km} / \mathrm{h}$ has been studied as the main parameter, the estimation of the channel based on the Ims algorithm has been proposed in order to improve the performance of the physical physical chain
\end{abstract}

\section{INTRODUCTION}

Short Range Communications Dedicated Receiver (DSRC) has been established in a $5.9 \mathrm{GHz}$ band [1]. For services involving vehicle-to-vehicle and vehicle-to-road communications. Even though the standard is not yet complete ,The DSRC system is one of the fundamental building blocks. The US Department of Transportation's "Vehicle Infrastructure Integration" (VII) initiative [2]. VII is considering a national system in which intelligent vehicles communicate with each other and with the transport infrastructure. The aim is to enable a number of new services that provide security, mobility and business benefits. In many ways, the deployment ofVII could reduce the number of fatalities on highways and improve the quality of life. The DSRC physical layer (IEEE 802.11p) [3] was originally adopted from the IEEE 802.11a [4] standard, which uses orthogonal frequency division multiplexing (OFDM) physical layer. Through the use of a guard interval, OFDM can mitigate inter symbol interference due to multipath fading. However, IEEE 802.11a was designed for stationary indoor environments with low propagation delay Wireless Access Vehicle (WAVE) environments involve dense urban centers, which tend to have significant delays spreads that would exceed the length of the guard interval in IEEE 802.11a. For this reason, the duration of the symbol was doubled, and so inherently the guard interval was also doubled from 0.8 to $1.6 \mathrm{~ms}$. Another major drawback of IEEE 802.11a is that it was designed for stationary environments. Conventional IEEE802.11a receivers initially estimate the channel response based on known preamble in the packet header. The channel response is assumed to be relatively static for the entire packet duration; therefore the entire packet is compensated based on the initial channel estimate [5]. The performance study in [6] indicated that conventional channel estimation is not a suitable choice for DSRC applications In order to improve the performance of DSRC systems, it's necessary to track the rapid fluctuation of the channel response within the packet duration. In this paper, a $5.9 \mathrm{GHz}$ DSRC receiver is designed to improve the performance in harsh vehicular channel conditions. In section 2, the current specifications of the DSRC physical layer are presented. Section 3 gives a brief overview of the channels. the Conventional environmental models are reviewed, Section 4 provides a description of the IEEE $802.11 \mathrm{p}$ physical coucje, Section 5 presents the simulation results which illustrate the performance improvements of the proposed DSRC receiver design over the conventional DSRC receiver. section 6 proposes the simulation of the LMS estimator of the channel as well as the results of the simulations as well as their interpretations section 7 concludes the paper

\section{II. . PHYSICAL LAYER SPECIFICATIONS}

The DSRC works with a physical layer based on OFDM. OFDM is a superior modulation technique for high speed wireless communications, which eliminates impacts of frequency selective fading channels. still used to implement digital audio broadcasting(DAB), digital video broadcasting (DVB), WLAN standardize 802.11a / g and HIPERLAN / 2) and new broadband Imax wireless access standard (IEEE 802.16).DSRC uses 64 subcarriers, 48 of which are dedicated to data and four are dedicated to pilot tones. These pilot tones Are spaced apart at $1.875 \mathrm{MHz}$ for phase tracking

\begin{tabular}{|c|c|c|c|c|c|c|c|c|c|}
\hline Short Preamble & & - & & & Ign & \multicolumn{4}{|c|}{ Data Symbols } \\
\hline $10 x$ & & & & & $8.0 \mu \mathrm{s}$ & & $8.0 \mu \mathrm{s}$ & & \\
\hline 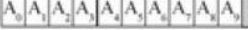 & $G_{\alpha}$ & $x_{v i}$ & $x_{v m}$ & $G$ & $x_{-m}$ & $G$ & $x_{1}$ & $\ldots$ & \\
\hline
\end{tabular}

Fig. 1 Transmission packet format

The packet format is shown in Fig. 1 with two preambles. The first preamble consists of ten short training symbols for packet detection; frequency offset estimation and symbol timing. The second preamble consists of two identical training symbols used for channel estimation (Train) subsequent to a long guard interval of length $\mathrm{G}_{\mathrm{CE}}=3.2 \mu \mathrm{s}$. The data rate is calculated by the following relation [1]

$R_{\text {data }}=\frac{\mathrm{NdsRc} \log 2 \mathrm{~m}}{\mathrm{Ts}}$

Where $\mathrm{m}$ is the number of modulation points in the mapping constellation, $\mathrm{Rc}$ is the code rate, Nods is the number of data subcarriers, and Ts is the OFDM symbol duration. For the DSRC physical layer, Ts and Nods are fixed at $8 \mathrm{~ms}$ and 48data subcarriers, respectively. Therefore the data rate is determined by selecting a transmission mode based on the digital modulation scheme and coding rate. IEEE $802.11 \mathrm{p}$ offers a data transmission rate of $3,4.5,6,9,12$, 18,24 and $27 \mathrm{Mbps}$ (Table 1) the frequency range used is 5.850 to $5.925 \mathrm{GHz}$, which is divided into $710 \mathrm{MHz}$ channels. The standard is based on four complex modulations: BPSK, QPSK, 16-QAM and 64-QAM. The modulation scheme used depends on the required data rate and the conditions of the wireless channel. OFDM is the technology of choice for high speed wireless communications. The transmission bandwidth is divided into many narrow sub-channels, which are transmitted in parallel. Ideally, each sub channel is narrow enough that the fade it experiences is flat. table 1 shows the comparison of phys in ieee $802.11 \mathrm{a}$ and ieee $802.11 \mathrm{p}$ ofdm allows the sub channels to overlap, which is spectrally efficient

Table 1 comparison IEEE 802.11 p AND IEEE 802.11 a

\begin{tabular}{|c|c|c|}
\hline Parameter & DSRC 802.11p & $802.11 \mathrm{a}$ \\
\hline $\begin{array}{c}\text { Information Data } \\
\text { Rate }\end{array}$ & $3-27 \mathrm{Mbps}$ & 6 -54 Mbps \\
\hline $\begin{array}{c}\text { Modulation Type } \\
\text { BPSK, QPSK, }\end{array}$ & BPSK, QPSK, \\
\hline $\begin{array}{c}\text { Coding Rate } \\
\text { Sumber of }\end{array}$ & $1 / 2,1 / 3,3 / 4$ & $1 / 2,1 / 3,3 / 4$ \\
\hline $\begin{array}{c}\text { OFDM Symbol } \\
\text { Duration }\end{array}$ & $\mathbf{5 2}(48+4)$ & $52(48+4)$ \\
\hline Guard Time & $1.6 \mu \mathrm{s}$ & $4 \mu \mathrm{s}$ \\
\hline FFT Periode & $6.4 \mu \mathrm{s}$ & $0.8 \mu \mathrm{s}$ \\
\hline Preamble Duration & $32 \mu \mathrm{s}$ & $3.2 \mu \mathrm{s}$ \\
\hline Bandwidth & $10 \mathrm{MHz}$ & $16 \mu \mathrm{s}$ \\
\hline Subcarrier & $0.156 \mathrm{MHz}$ & $20 \mathrm{MHz}$ \\
\hline
\end{tabular}




\begin{tabular}{|c|c|c|}
\hline $\begin{array}{c}\text { Frequency Spacing } \\
\text { Parameter }\end{array}$ & DSRC 802.11p & $802.11 \mathrm{a}$ \\
\hline $\begin{array}{c}\text { Information Data } \\
\text { Rate }\end{array}$ & $3-27 \mathrm{Mbps}$ & $6-54 \mathrm{Mbps}$ \\
\hline $\begin{array}{c}\text { Modulation Type } \\
\text { Coding Rate }\end{array}$ & $1 / 2,1 / 3,3 / 4$ & $1 / 2,1 / 3,3 / 4$ \\
\hline $\begin{array}{c}\text { Number of } \\
\text { Subcarriers }\end{array}$ & $52(48+4)$ & $52(48+4)$ \\
\hline $\begin{array}{c}\text { OFDM Symbol } \\
\text { Duration }\end{array}$ & $8 \mathrm{QS}$ & $4 \mu \mathrm{s}$ \\
\hline Guard Time & $1.6 \mu \mathrm{s}$ & $0.8 \mu \mathrm{s}$ \\
\hline FFT Periode & $6.4 \mu \mathrm{s}$ & $3.2 \mu \mathrm{s}$ \\
\hline Preamble Duration & $32 \mu \mathrm{s}$ & $16 \mu \mathrm{s}$ \\
\hline Bandwidth & $10 \mathrm{MHz}$ & $20 \mathrm{MHz}$ \\
\hline & & $16 \mathrm{QAM}, 64 \mathrm{QAM}$ \\
\hline Subcarrier & $0.156 \mathrm{MHz}$ & $0.3125 \mathrm{MHz}$ \\
\hline Frequency Spacing & & \\
\hline TABLE 2 requirr & & \\
\hline
\end{tabular}

TABLE 2 requirements off IEEE 802, $11 \mathrm{p}$

\begin{tabular}{|c|c|c|}
\hline Phi parameter & $\begin{array}{c}\text { Relevant channel } \\
\text { parameters }\end{array}$ & $\begin{array}{c}\text { Criteria for phi } \\
\text { parameter }\end{array}$ \\
\hline $\begin{array}{c}\text { guard interval } \\
\text { (GI) }\end{array}$ & $\begin{array}{c}\text { Maximum excess } \\
\text { delay (be) }\end{array}$ & GI $>($ be $)$ \\
\hline $\begin{array}{c}\text { Carrier spacing } \\
\text { Do }\end{array}$ & $\begin{array}{c}\text { Coherence band } \\
\text { with }(\text { Bc) } \\
\text { Doppler spread } \\
\text { BD }\end{array}$ & BC> Do $>$ BD \\
\hline $\begin{array}{c}\text { Interval between } \\
\text { channel } \\
\text { estimates (packet length } \\
\text { Top) }\end{array}$ & $\begin{array}{c}\text { Coherence time } \\
\left(T_{c}\right)\end{array}$ & Top $\left.<T_{c}\right)$ \\
\hline
\end{tabular}

TABLE 3: summry devices of different envirronement in DSRC

\begin{tabular}{|c|c|c|c|}
\hline $\begin{array}{c}\text { ENVIRONN } \\
\text { EMENT }\end{array}$ & $\begin{array}{c}\text { SEPARA } \\
\text { TION } \\
(\mathbf{m})\end{array}$ & $\begin{array}{c}\tau \mathrm{rms} \\
(\boldsymbol{\mu s})\end{array}$ & $\begin{array}{c}\mathbf{D}_{\mathbf{s}} \\
(\mathbf{H Z})\end{array}$ \\
\hline Highway & $0-50$ & 21.09 & 567.88 \\
LOS & $50-200$ & 95.02 & 2714.29 \\
& $200-500$ & 112.33 & 1520.39 \\
& $>500$ & 50.88 & 1248.61 \\
\hline Highway & $0-150$ & 382.01 & 382.01 \\
NLOS & $150-300$ & 3750.94 & 3750.94 \\
& $>300$ & 3289.93 & 3289.93 \\
\hline Urban & $0-75$ & 10870.13 & \\
LOS & $75-150$ & 3347.11 & 1270.35 \\
& $150-250$ & 5232.32 & 2309.40 \\
& $250-350$ & 3188.55 & 2309.40 \\
& $350-450$ & 3396.36 & 1700.61 \\
& & 10933.88 & 1700.61 \\
\hline Urban & $0-150$ & 1739.39 & \\
NLOS & $150-250$ & 2174.24 & 2374.21 \\
& $250-500$ & 2370.61 & 950.23 \\
& $500-800$ & 2227.27 & 1146.29 \\
& $800-1000$ & & 491.92 \\
& & & \\
\hline Rural & $0-120$ & 3132.87 & 783.59 \\
LOS & & & \\
\hline & & & \\
\hline
\end{tabular}

III. V2V CHANNEL

Multipath causes the propagation of radio signals transmitted from one antenna to another through two or more paths. In urban areas, fading arises due to the different height of the transmitting and receiving antennas as there is no particular line of sight (NLOS) to propagate the signal from one antenna to another [7]. But fading may happen even there is a presence of a Line of Sight (LOS) due to the reflection, scattering etc. of the transmitted signal from the ground and surrounding area objects. The receiving antenna will get a resultant signal which can vary widely in amplitude or even in phase based on the distribution of the intensity and the bandwidth of the transmitted signal. The amplitude variations of multipath fading signals are followed by Rayleigh and Rician distributions. Multipath propagation can be categorized as large scale and small scale fading. Small scale fading can be expressed as rapid fluctuations of the amplitude or phase of the transmitted radio signal over a very short period of time or short travel distance. Large scale fading is the consequence of signal attenuation due to the propagation over long distances and diffraction around large objects. Multipath small scale fading effect causes few rapid changes in radio channels media such as rapid changes in signal strength level, time dispersion produced by multipath propagation path delays and random frequency modulation due to varying Doppler shifts of different multipath signals [8]. The small scale fading can be expressed as a linear filter with the time varying impulse response of a wireless channel. Time variation is introduced due to the receiver motion in space and filtering nature is introduced due to the summation of amplitudes, delays of multiple arriving waves at any instant of time. However based on the time delay spread small scale fading can be categorized as flat fading and frequency selective fading. As per Doppler spread small scale fading can be categorized

\section{1. wave channel}

A wireless medium between two Omni-directional antennas is usually made up of a secular component, namely line-of-sight (LOS) and scatter (non-LOS) components. The multipath channel is characterized by the power delay profile, which is used to obtain the delay spread. The movement of the vehicle introduces a time varying channel in addition to the multipath fading. The time varying nature of the channel is quantified by the coherence time, $T_{c}$ which is obtained from the relation [2] $T_{c}=\frac{1}{2 f_{d}}=\frac{c}{2 v f_{c}}$ (2) where $\mathrm{v}$ is the relative velocity in meters per second $(\mathrm{m} / \mathrm{s})$, fc is the carrier frequency in Hertz $(\mathrm{Hz})$, and $\mathrm{c}$ is the speed of light in $\mathrm{m} / \mathrm{s}$. The WAVE channel can be modeled using statistical models presented in [9]. Rayleigh fading channels represent 2D isotropic scattering environments without a LOS component. Under Rayleigh fading, the received complex envelope is treated as a wide-sense stationary Gaussian random process with zero mean. When a LOS component exists, the envelope becomes a non-zero mean Gaussian random process. This type of fading is called Rican fading, where the Rice factor, K, is defined as the ratio of the secular to sum of scatter power. According to [10], a wireless channel can be considered static over a period of $\mathrm{T}$ if $\mathrm{T} \ll T_{c}$. Therefore at a $5.9 \mathrm{GHz}$ band, the channel could have static characteristics over the entire packet duration, Top, only if it satisfies the following relation $\mathrm{Tp}=\mathrm{N}_{\mathrm{s}} \mathrm{T}_{S}<<\frac{0.0215}{\mathrm{v}}$ (3) where $\mathrm{Np}$ is the number of OFDM data symbols per packet. In (3), if any one of the three parameters (Stun, or v) is increased while holding the others constant, the rate of fading also increases. Fig.3 illustrates different Rayleigh fading envelopes produced by the channel simulator. Each plot is a random example of possible fading envelopes across one packet in wireless communication multipath fading is an unavoidable phenomenon, an information carrying signal transmitted by the channel having BT bandwidth. The channel is said to have frequency selective fading if by is less than BT, it is said to be non-frequency selective or to uniform fading if by is greater than BT the consistency band is defined by: $B_{c}=\frac{1}{5 c_{r m s}}=$ $\frac{1}{c_{\max }}$ where $r_{\max }$ and $r_{\text {rms }}$ are the spread and the maximum of the delay propagation delay, respectively. $r_{\max }$ Is defined to be less than the upper value propagation with limited delay $r_{l i m}=T_{G I}=1.6 \mu$ s the coherence time of the channel event is linked to the vehicle speed $\mathrm{v}$ by $T_{c}=\frac{1}{2 f_{d}}=\frac{c}{2 v f_{c}}$ where FD is the Doppler frequency, face is the carrier frequency, $\mathrm{c}$ is the speed of light and $v$ is the speed of the vehicle. A slowly changing channel has a small Doppler frequency or, equivalently, a large coherence time. If the coherence time $T_{c}$ is small, compared to the measured time interval Tm of the transmitted signal, the channel is said to be fast fading. Otherwise, the channel is said to be slow fading. The channel parameters include BT, Tm and slim. BT $=10 \mathrm{MHz}$; the measured signal interval $\mathrm{Tm}=$ packet time $=840$ as; each transmitted packet contains 100 orthogonal frequency division multiplexing (OFDM) symbols; the maximum delay spreading time mix is set to be less than the guard interval (GI) TGI of the OFDM symbol, i.e. the limit of the maximum delay spreading time slim = $\mathrm{TGI}=1.6 \mu \mathrm{s}$. Fig. 3 illustrates different Rayleigh fading envelopes produced by the channel simulator. Each plot is a random example of possible fading envelopes across one packet duration of $0.8 \mathrm{~ms}$, with a packet length, $\mathrm{Np}$, of 100 data symbols per packet. At high velocities, deep fades may occur, which practically eliminate the signal. As velocity is increased, the number of deep 
fades per packet will also increase. Appendix 3 gives matlab program

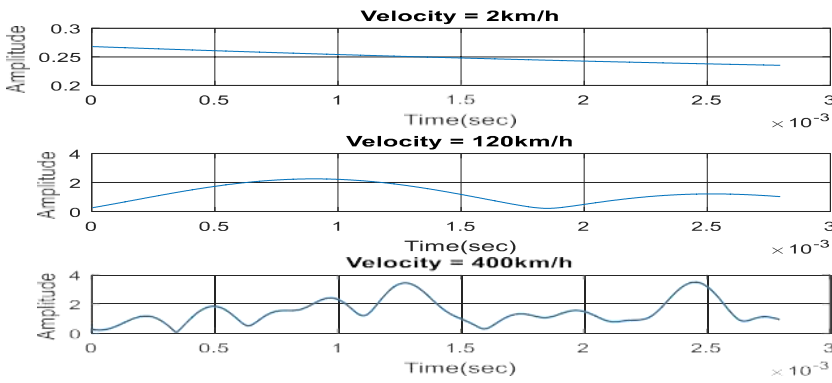

Fig. 3 Examples of fading envelopes for 5.9 GHz DSRC

( Ts à $0.8 \mathrm{~ms}$ ) over a packet duration of $0.8 \mathrm{~ms}$ (Nap 100)

\subsection{Environment comparisons}

With regards to the proposed DSRC standard, examining Table 2 suggests that the proposed design parameters of $802.11 \mathrm{p}$ should account for many, but not all, of the channel impairments. We start by comparing the guard interval with the delay spreads to determine the degree to which multipath will introduce ISI. For $802.11 \mathrm{p}$, this guard interval (GI) is $1.6 \mu \mathrm{s}$, which is twice as long as 802.11a's GI of $0.8 \mu$ s. Among the high-speed environments (LOS / NLOS highway and rural), we see that the rural environment is the least hostile among the three due to its low delay.spread resulting from a lack of diffusers. closely followed by the LOS motorway environment, which has higher average excess delays ( $32 \mathrm{~ns}$ more) but Lower RMS delays ( $91.23 \mathrm{~ns}$ less) Short distance (0 to $50 \mathrm{~m}$ ), LOS highway the channels have the most favorable performance compared to the others in case of delay and the Doppler characteristic are both taken into account. On the other hand, the NLOS motor way the performance of the channels is much worse. Although the average and RMS delays exceed the LOS highway equivalents of only $151 \mathrm{~ns}$ and $184 \mathrm{~ns}$, respectively, Doppler the diffusion is much higher at $3515.03 \mathrm{~Hz}$, i.e. a gain of $2291 \mathrm{~Hz}$ on the LOS highway equivalent an examination of Table 3 shows that all environments should have coherence bandwidths larger than the spacing between pilot tones. If the figure of merit is changed to sum delay spread, however, only the highway LOS and rural LOS environments consistently have a sufficiently wide coherence bandwidth. Highway NLOS has an average sum delay spread of $507.02 \mathrm{~ns}(1.972 \mathrm{MHz})$, while urban LOS/NLOS environments are at $748.91 \mathrm{~ns}(1.335 \mathrm{MHz})$ and $704.9 \mathrm{~ns}(1.419 \mathrm{MHz})$, Table 3 shows that all environments should have higher consistency bandwidths as the spacing between pilot tones. If the merit number is replaced by a sum delay However, only propagate highway LOS and rural LOS environments consistently have a sufficiently large coherence bandwidth. Highway NLOS has an average sum delay propagation of $507.02 \mathrm{~ns}(1.972 \mathrm{MHz})$, while urban LOS / NLOS environments are at748.91 ns (1.335 MHz) and 704.9 ns (1.419 $\mathrm{MHz}$ ), static environments in which 802.11 systems often operate

\section{CONVENTIONNEL SYSTEM}

Figure 4. gives the diagram of the transmitter. Forward Error Correction (FEC) coding is used to detect and correct errors äte to channel fading and noise. The FEC code used in this system [10] is convolutional at $1 / 2$ code rate with a generator (1338, 1718 ) and a constraint length of 7. Figure 4: Comparison of IEEE 802.11 a and IEEE 802.11 p with BPK BER versus SNR in one WAVE channel, deep n one WAVE channel, deep fading can cause a long error sequence, which can make the decoder inefficient. In order to alleviate symbol correlation, the bits are scrambled with a block interleaver. The length of the block interleaver corresponds to an OFDM data symbol The size of the block depends on the scheme selected by the modulations The digital modulation schemes scheme selected by the modulations The digital modulation schemes available include gray-coded constellations of binary phase shift modulation, phase shift modulation in quadrature (QPSK), quadrature amplitude modulation of 16 zones (16-QAM) and 64-QAM. The interleaved bits are digitally modulated and divided into 48 subchannels with four fixed pilot tones denoted $X_{n, k}$. the parallel data is then multiplexed into a 64-point inverse fast Fourier transform (IFFT) [4]. The IFFT version, $\mathrm{xn}, \mathrm{k}$, is then converted to high rate serial data $\left(x_{i}\right)$. Finally, the guard interval is inserted to produce $X_{I G}$. At the receiver, the guard interval is removed from the received signal, The parallel data, $y_{I G} \mathrm{yn}, \mathrm{k}$, is demultiplexed in the FFT, producing the following output in the frequency

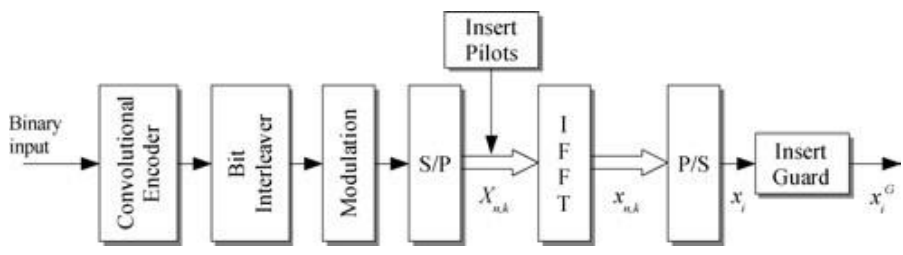

Fig. 4.a:transmitter in ieee $802.11 \mathrm{p}$

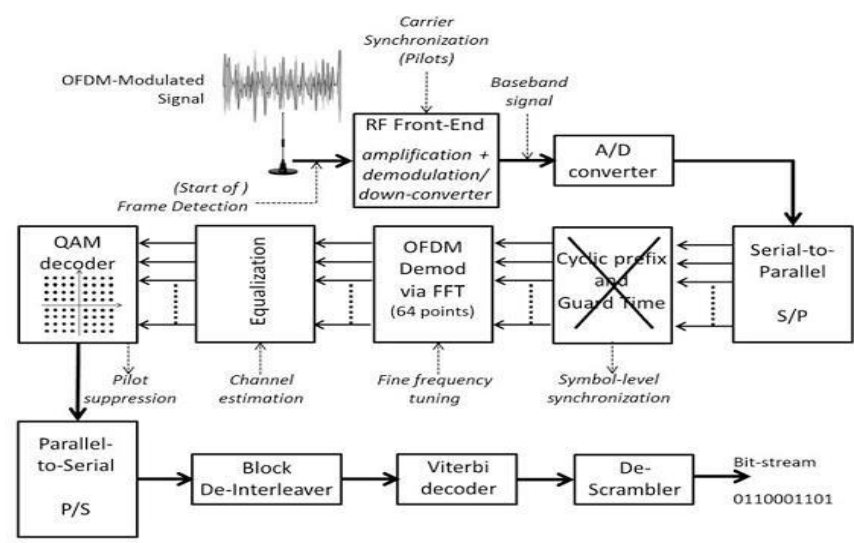

Fig. 4 b:receiver in ieee $802.11 p$

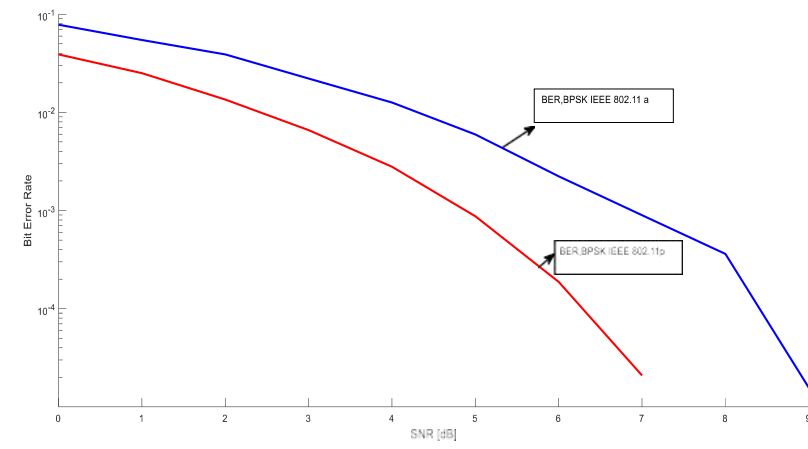

Fig. 5 comparing IEEE $802.11 \mathrm{a}$ and IEEE $802.11 \mathrm{p}$ with BER versus SNR BPSK

\subsection{Comparing IEEE 802.11 p AND IEEE 802.11 a}

The latest technologies, such as $802.11 \mathrm{p}$, are based on OFDM PHY, as OFDM based transmission systems can provide high speed transmission and high spectral efficiency in channel environments. Fig. 4.1: The OFDM $802.11 \mathrm{p}$ system model. The configuration of the model prepared in accordance with the recommendations of the IEEE 802.11a standard [87]. A simulation model was implemented using MATLAB. The structures of transmitters and receivers for $802.11 \mathrm{p}$ and $802.11 \mathrm{a}$ correspond to the blocks in figure 4 The simulation model includes the following signal processing steps: transmitter, channel, receiver. The transmitter:

- Convolutional coding with a coding rate, $R=1 / 2$ and $R=3 / 4$;

- BPSK modulation scheme; • Inverse Fast Fourier Transform (IFFT) for 64 samples;

- Addition of a cyclic prefix (CP) of a length of a quarter of the duration of the symbol; The channel:

- Signal transmission with a duration of $8 \mu \mathrm{s}$ (for 802.11p) and $4 \mu \mathrm{s}$ (for 802.11a) on the fading channel HIPERPLAN / 2; The receiver:

- Removal of the CP

- Fast Fourier transform (FFT) for 64 sub-carriers

- Estimation of the least mean squares (LMS) channel;

- BPSK demodulation

- Decoding of error codes using Viterbi's hard decision algorithm. To obtain precise results, ten thousand bits are transmitted. More Data 
transmission was simulated to vary $E b$ / $N 0$ on Rayleigh fading channels: model A, shown in Figures 6, two BER curves were obtained: for IEEE 802.11a and IEEE 802.11p standards. For low values of mean $E b / N 0$, the errors are the same. The mean $E b / N 0$ required for a BER of $10-4$ is $2 \mathrm{~dB}$ we notice the weak ber that presents ieee 802.11 p compared to ieee 802.11 a

\section{MATLAB SIMULATION OFDM IEEE 802.11 P}

\section{Doppler shift in IEEE $802.11 \mathrm{p}$}

Doppler shift when a source vehicle and a receiver vehicle move relative to each other, the frequency of the received signal will not be the same as the source. [13] When they get closer to each other, the frequency of the received signal is higher than the source, and it decreases when they get closer as seen in [9]. The Doppler effect has a great effect on vehicle networks due to the VANET mobility characteristic the frequency Doppler shift can be calculated $\Delta \boldsymbol{f}= \pm \boldsymbol{f}_{\boldsymbol{c}} \frac{v}{c} \cos \mathrm{Q}(1), \Delta \boldsymbol{f}$ where is the deviation of the source vehicle frequency at the receiver. The frequency of the source is $\boldsymbol{f}_{\boldsymbol{c}}, \boldsymbol{v}$ is the speed difference between the source and transmitter vehicles, and $\mathrm{c}$ is the light velocity. In vehicular networks the range of $f_{c}=5.9 \mathrm{GHZ}$ for simulation we choose $\mathrm{v} \in[25,400 \mathrm{~km} / \mathrm{h}]$ wich give $\Delta f \in[136,2185,2 \mathrm{~Hz}]$ for Doppler shift,.. OFDM symbol is very sensitive to the Doppler Shift. An OFDM signal can be represented as follows : $\boldsymbol{V}_{(t)}=\sum_{i=1}^{N_{s}-1} \boldsymbol{A}_{i} \cos \left(m_{i} \boldsymbol{t}+\boldsymbol{\varphi}_{i}\right)$ (2) with the $A_{i}, \omega_{i}, \varphi_{i}$ are the respective values of the amplitude, angular frequency, and phase of subcarrier used from the it to OFDM signal. If Ns are the number of subcarriers used in OFDM, then each subcarrier must be orthogonal to one another. This can be obtained if $f_{i}=\underline{\omega} i$ which is the integer multiplication of $\frac{1}{2 T}$, with $\mathrm{T}$ is the period of the data, and $\mathrm{f} 1$ is the frequency range with a value of $R_{s}=\frac{1}{T}$.If we continued equation (2) with processing at up converter side to produce 5,9 GHz frequency, (2)becomes, $V_{i}=A_{i} \cos \left(\omega_{i} t+\varphi_{i}\right) e^{-\left(j 2 \pi f_{c}\right) c_{i}}$ if he value $A_{i} \cos \left(\omega_{i} t+\varphi_{i}\right)=S_{i}$ then $\boldsymbol{V}_{\boldsymbol{i}}=\boldsymbol{S}_{i} \boldsymbol{e}^{-(j 2 \pi f c) r_{i}}(\mathbf{3})$ When the value of $e^{-(j 2 \pi f c)}$ and $r_{i}$ represents the carrier frequency and delay to the it component of the received signal, respectively. If the vehicle moves at a constant velocity $\mathrm{v}$, and each vehicle moves straight with $\beta=0$ then the value of the transmitted signal is affected by the value of $\frac{v}{c} \cos Q$ so the equation (3) become $\boldsymbol{V}_{\boldsymbol{i}}=\boldsymbol{S}_{\boldsymbol{i}} \boldsymbol{e}^{-(j 2 \pi f c) r_{\boldsymbol{i}}} \boldsymbol{e}^{(j 2 \pi \Delta f) t}$ with $(j 2 \pi \Delta f) t$ is a value from phasechanging at the transmitted signal. If the value of $t=1 / 4 \Delta f$, then the equation becomes $V_{i}=j e^{-\left(j 2 \pi f_{c}\right) c_{i}}$ Assuming the period is $1 / 4$ of the Doppler shift $0 \leq$ $\Delta f \leq \pm 2.185 \mathrm{kHz}$,

MATLAB simulation model

the MATLAB simulation model see APPENDIX 1 is made for a vehicle speed variation of 25.75,125 175225 and $400 \mathrm{~km} / \mathrm{h}$, the SNR varies from 1 to $40 \mathrm{~dB}$ Figure 2 gives the result of parameters as defined by the IEEE 802.11a is between 0 and $250 \mathrm{~km} \mathrm{/} \mathrm{h,} \mathrm{therefore} \mathrm{the} \mathrm{amount} \mathrm{of} \mathrm{frequency} \mathrm{deviation} \mathrm{varies} \mathrm{between}$ approximately $\pm 7.708 \mathrm{kHz}$. The amount of offset can be very small, however, this offset causes significant problems in PHY transmission because the transmission technique (OFDM) is very sensitive to carrier frequency offset In IEEE 802.11p, the subcarrier spacing has been halved because the WAVE OFDM receiver is more sensitive to carrier frequency offset and Doppler shift. V2V and V2I communications are sensitive to much faster fading and higher Doppler frequency propagation and wave propagation. Multi-path delay higher than any other wireless system. In addition, it must be extremely robust in abnormal situations as collisions and accidents rarely occur under normal conditions. ? Depending on the limitation of the $802.11 \mathrm{p}$ standard, the channel used is a combination of a Rayleigh and an AWGN. For BER 10e-3 value if the moving speed is $30 \mathrm{~km} / \mathrm{h}$. no communication can be made when the SNR is less than $10 \mathrm{~dB}$; for communication to be effective at $\mathrm{SNR}=16 \mathrm{~dB}$, the maximum relative speed must be less than or equal to $175 \mathrm{~km}$ / h. Figure 6a describes the impact of the Doppler effect in the IEEE $802.11 \mathrm{p}$ standard due to the high mobility of the channel, it is observed that the BER decreases if the speed increases which discourages transmission

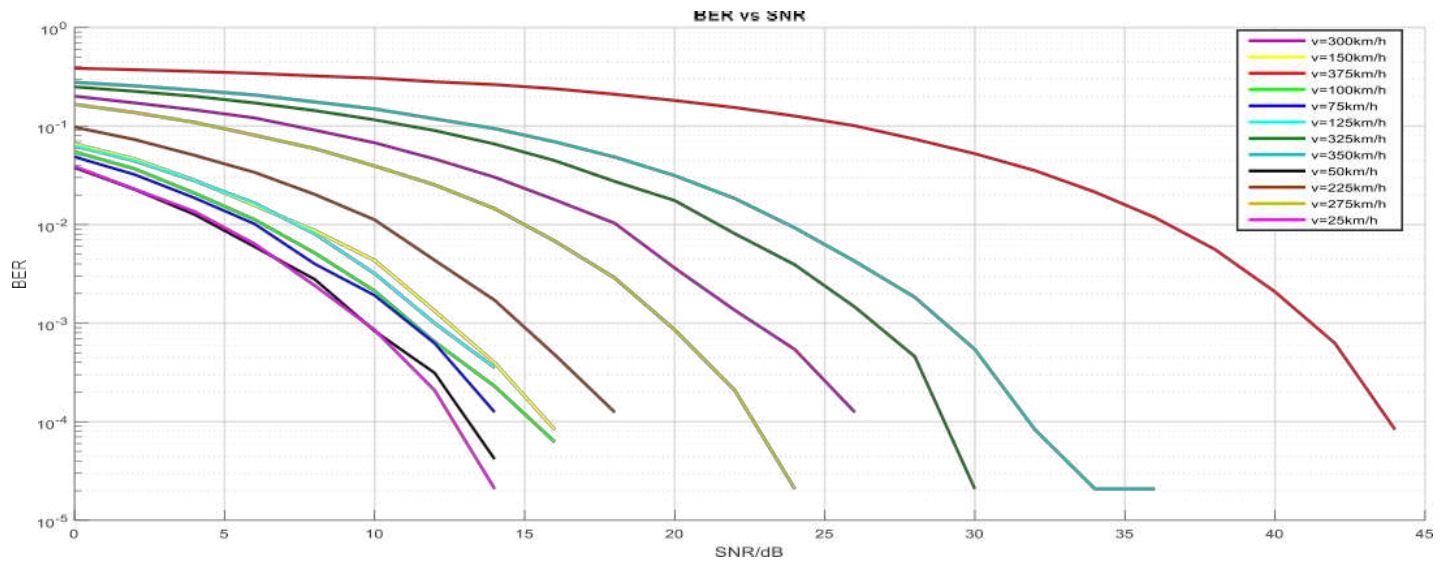

Fig. 6. a BER BPSK. V є [ $25,400 \mathrm{~km} / \mathrm{h}]$
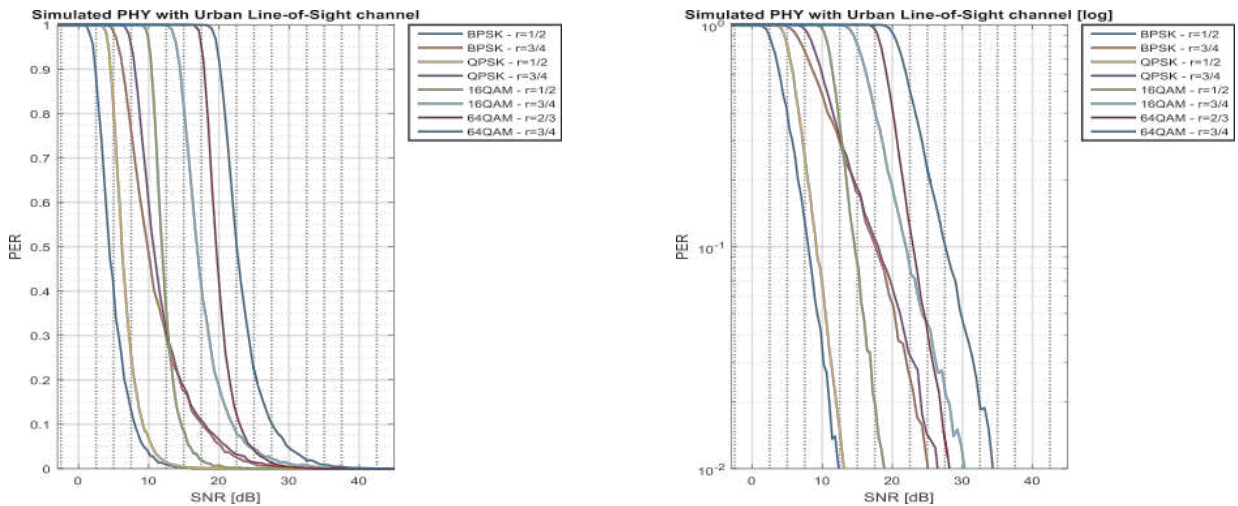

Fig. 6.b ber for differents types modulation and ( code K) 
Figure $6 \mathrm{~b}$. gives a Per (packet error rate) as a function of the SNR for different types of off modulations and a different value of code $K$, we conclude that the BPSK modulation and $\mathrm{K}=1 / 2$ gives the best performance (ber the most) which is why these parameters have been fixed in all the previous matlab simulations In order to give a realization of the physical layer of the ieee $802.11 \mathrm{p}$ similar to the ieee 802.11 layer a the program given in appendix 2 performs this task, the encoding process consists of of several steps, the transmitted bits are generated with the data source component. These data are modulated using phase shift modulation (BPSK or QPSK) or amplitude modulation (16-QAM or 64 QAM) the transmitted signal rate which can vary by $3 \mathrm{Mb} / \mathrm{s}$ (with BPSK and $1 / 2$ code speed) at $27 \mathrm{Mb} / \mathrm{s}$ (with 64QAM and code rate 3/4. Figure 7 and figure 8 compare the modulation, $\mathrm{M}$ ary, we find there respectively the BER the qam 4 and the qam 64, that we can see that 4 QAM has a low BER compared to 64 QAM Figures 9 and 10 respectively give the constellations which are the distance between the points I and the modulation system $\mathrm{Q}$ for $\mathrm{M}=4$ and $\mathrm{M}=644$-QAM was found to provide the best performance (lowest BER) compared to 16-QAM and 64QAM. The comparison results also included a modulation scheme with a lower constellation value at better BER performance due to the higher bit rate, we note the influence of the Rayleigh-type channel as in the form of noise on the constellation of modulations $\mathrm{We}$ also validate the communication performance of OFDM systems in summary, the simulations see the negative effect of the Doppler effect on the performance of the transmission, namely he degradation of the cradle when the speed of the mobiles increases Table 5 summarizes the situations channel operation

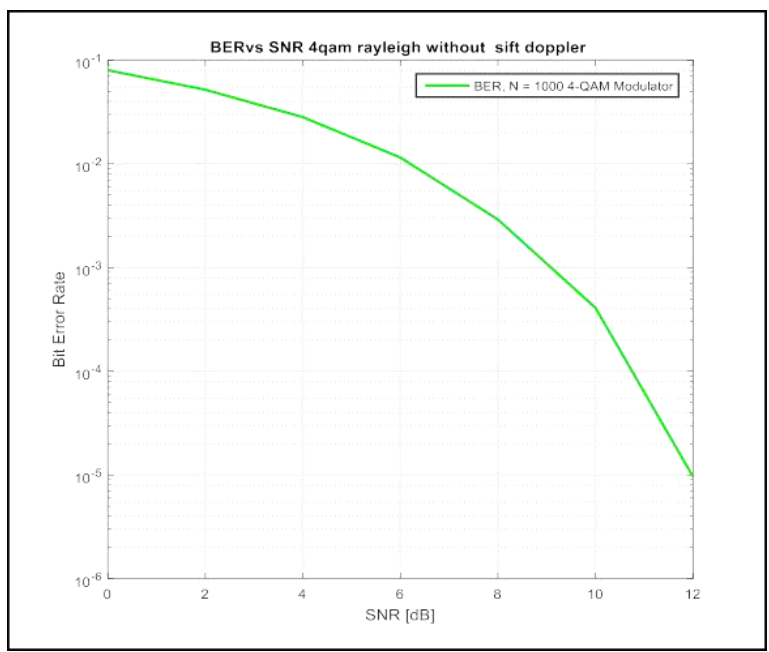

Fig. 7 . BER Vs SNR 4QAM

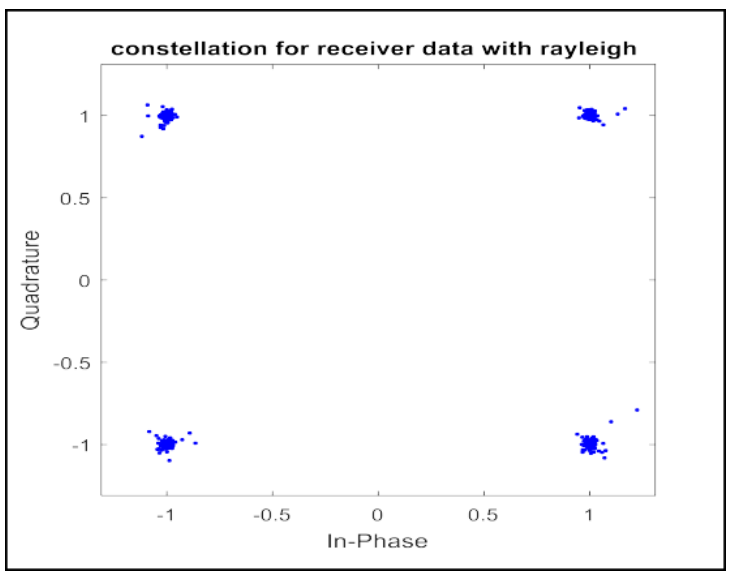

Fig. 9 received constellation 4 QAM
Table 5: resultats simulation fading in wave channel

\begin{tabular}{|c|c|c|}
\hline & FLAT FADING & $\begin{array}{c}\text { FREQUENCY SELECTIVE } \\
\text { FADING }\end{array}$ \\
\hline SLOW FADING & $0 \leq r_{R M S} \leq 20 \mathrm{nsec}$ & $20 \mathrm{nsec} \leq r_{R M S} \leq 320 \mathrm{~ns}$ \\
& $0 \leq f_{d} \leq 595,2 \mathrm{~Hz}$ & $0 \leq f_{d} \leq 595,2 \mathrm{~Hz}$ \\
& $0 \leq \mathrm{v} \leq \mathrm{v} \leq 109 \mathrm{~km} / \mathrm{h}$ & $0 \leq \mathrm{v} \leq \mathrm{v} \leq 109 \mathrm{~km} / \mathrm{h}$ \\
\hline FAST FADING & $0 \leq r_{R M S} \leq 20 \mathrm{nsec}$ & $20 \mathrm{nsec} \leq r_{R M S} \leq 320 \mathrm{~ns}$ \\
& $595,2 \leq f_{d} \leq 595,2 \mathrm{H}$ & $595,2 \leq f_{d} \leq 2185,2 \mathrm{~Hz}$ \\
& $109 \mathrm{~km} / \mathrm{h} \leq \mathrm{v} \leq 400 \mathrm{~km} / \mathrm{h}$ & $109 \mathrm{~km} / \mathrm{h} \leq \mathrm{v} \leq \mathrm{v} \leq 400 \mathrm{~km} / \mathrm{h}$ \\
& & \\
& & \\
& & \\
& & \\
& & \\
& & \\
& &
\end{tabular}

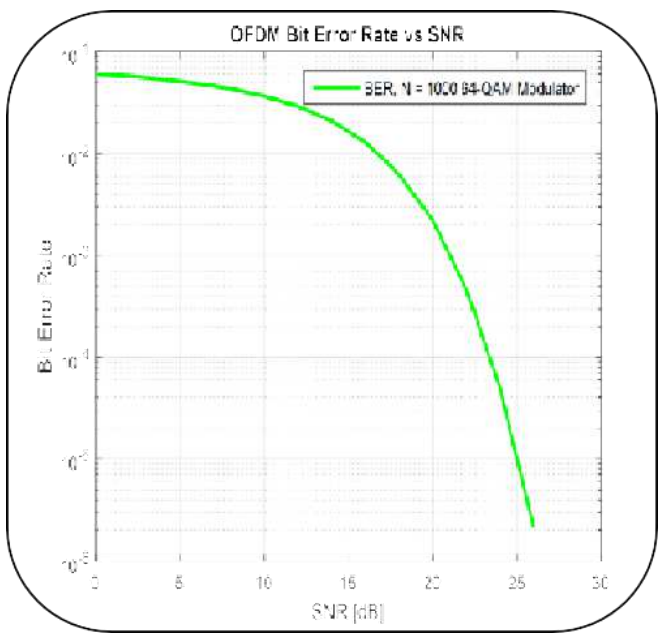

Fig. 8. BER V S SNR 64QAM

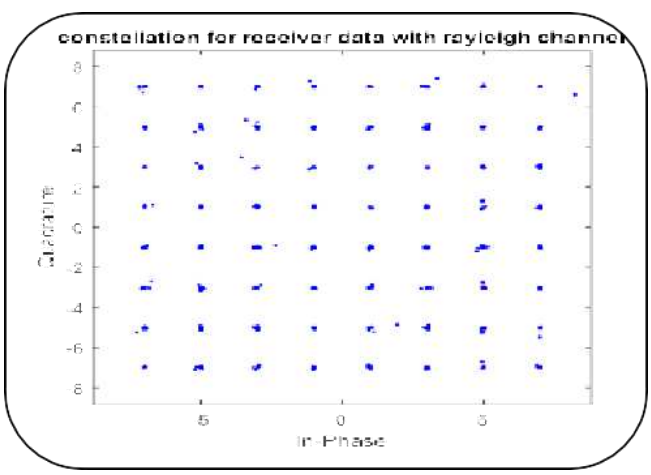

- Fig. 10. received constellation 64 QAM 


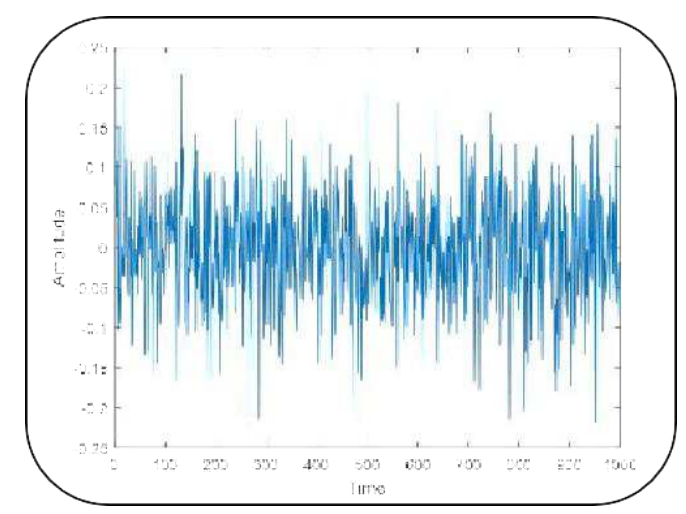

Fig. 11 (a) time domain waveform of $O F D M$ in $B P S K$

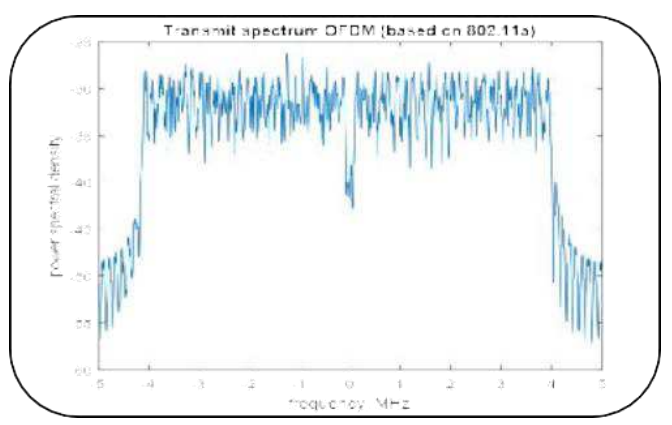

Fig. 11 (b) . represents the power spectrum density

\section{ADAPTIVE FILTERING (AF) FOR}

\section{CHANNEL EQUALIZATION}

. Adaptive Filtering (AF) describes how it has been shown to be an effective filter in channel equalization using self-adjusting channel impulse response coefficients (CIR) based on an adaptation algorithm such as least mean squares (LMS), recursive least squares (RLS) and Kalman (KF) filtering. The LMS algorithm is a simple algorithm among them to implement it practically with less computation cost and it changes the CIR coefficients (h (n)) with an effort to reduce the mean square error (MSE) which is the cost of the function. Different variations of the adaptive LMS algorithm such as Fixed Step Size a) LMS (FSS), Variable Step Size LMS (TVS) and Adaptive Step Size LMS b) parameter [14]. A. Variants of LMS Algorithms FSS-LMS Algorithm The LMS algorithm for updating the CIR coefficients is as follows.

$\mathbf{y}(\mathbf{n})=)=h^{T}(\mathbf{n}) \mathbf{x}(\mathbf{n})(\mathbf{4})$

e $(n)=d(n)-y(n)(5)$

$h(n+1)=h(n)+\mu e(n) u(n)(6)$

where $x(n)$ is the input of the channel, e (n) is the error calculated by the AF, $\mathrm{d}(\mathrm{n})$ being the desired output and $\mu$ is the convergence parameter or the step size for the update. day of the CIR coefficients which decides the stability of the mathematical equation described for the update of the CIR coefficients can be better explained by the block diagram which is shown in Fig.11

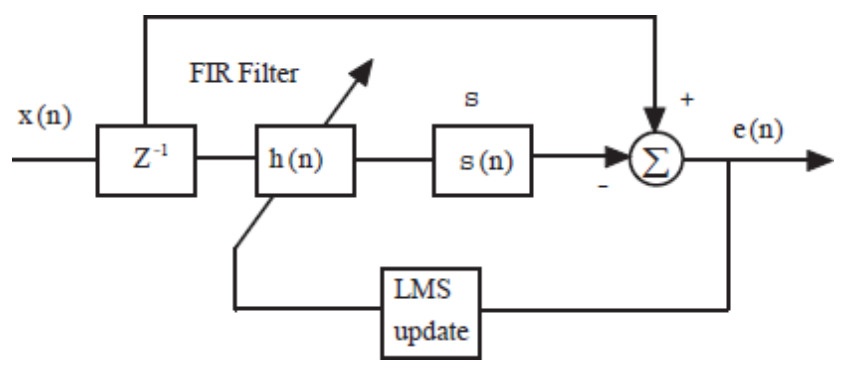

Fig. 11 adaptatif filtre lms

a matlab script for simulation isgiven in appendix 4

. ASS-LMS AF for udating CIR filter coefficints with inclusion of gradient vector, which is basis for improvement in convergence rate, adaptibility made a way to rapid varying channels ease of channel estimation and equalization.

Convergance Analysis of FSS-LMS came from the adatation in step size with inclusion of gradient vector

To evaluate the performance of the proposed ASS-LMS the convergence analysis of different step size algorithms are evaluated with reference to number of iterations as shown in Fig. 12. the effect of the pitch (mu) on the constellation of the signal received from the physical chainis shawen in fig 13

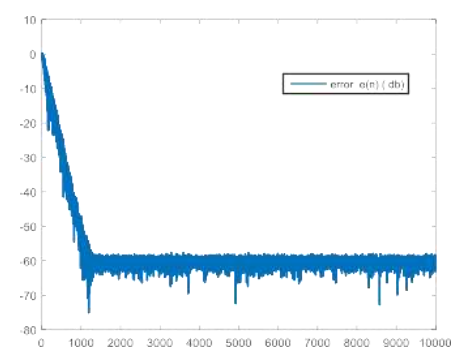

Fig. 12: error in Ims algorithm

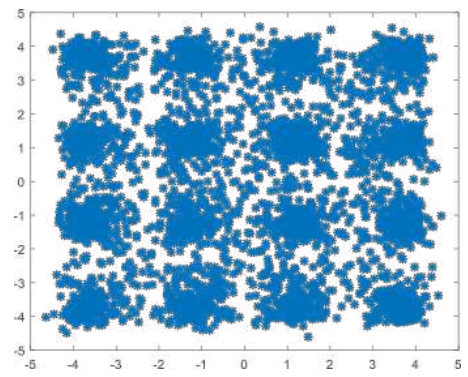

a) constellation for received signal for $\mu=0.00001$

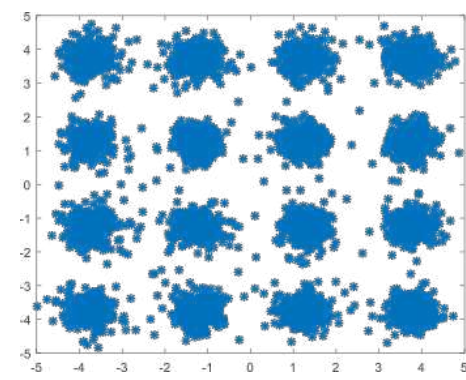

b ) constellation for received signal for $\mu=0.0001$

Fig. 13 constellation for received signal

\section{CONCLUSION}

While much emphasis has and will deservedly be given to DSRC as amechanism for increasing safety and efficiency on public roadways, its viability rests on the usage of a robust, low-latency physical layer. Decisions regarding DSRC's projected benefits should always take into account the capabilities of its physical layer technology and the ground truth of the environment under which it operates.

Measurements of this ground truth, by the authors and others, have shown the current DSRC standard to be sufficient, but not necessarily optimal, for its intendedenvironment. Although the proposed standard may perform acceptably for short transmissions, longer transmissions may be subject to higher error rates in the absence of further processing. Analysis of our measurements also suggests the need to examine topics such as reduced coherence bandwidths, power control, and angleof-arrival. All of these items impart challenges that will affect higher layers of the DSRCprotocol stack. Conversely, their presence also provides fertile ground for further research and innovation. The authors hope that this chapter has brought sufficientattention to these issues and has encouraged readers to further appreciate how physical layer issues play a driving role in DSRC's capabilities and future applications.

References

[1] http://grouper.ieee.org/groups/scc32/dsrc, accessed September 2006

[2] Fazel, K., \& Kaiser, S. (2008). Multi-carrier and spread spectrum systems: from OFDM and MC-CDMA to LTE and WiMAX. John Wiley \& Sons.

[3]. http://grouper.ieee.org/groups/802/11/Reports

[4]. IEEE Std. 802.11a: 'Wireless LAN medium access control (MAC)

[5]. Lu, J., Letaief, K. B., Chuang, J. I., \& Liou, M. L. (1999). M-PSK and M-QAM BER computation using signal-space concepts. IEEE 
Transactions on communications, 47(2), 181-184.

[6]. Abdulhamid, H., Tepe, K., and Abdel-Raheem, E.: 'Performance of DSRC systems using conventional channel estimation at high velocities', Int. J. Electron. Commun. (AEU“*), 2007, 61, pp. 556-561

[7] Sun, M. T., Huang, L., Wang, S., Arora, A., \& Lai, T. H. (2003). Reliable MAC layer multicast in IEEE 802.11 wireless networks. Wireless Communications and Mobile Computing, 3(4), 439453.

[8 ]Durgin, G. D. (2003). Space-time wireless channels. Prentice Hall Professional.

[9] Babu, A. S., \& Rao, D. K. S. (2011). Evaluation of BER for AWGN, Rayleigh and Rician fading channels under various modulation schemes. International Journal of Computer Applications, 26(9), 23-28.

[10] Cavers, J. K. (1991). An analysis of pilot symbol assisted modulation for Rayleigh fading channels (mobile radio). IEEE transactions on vehicular technology, 40(4), 686-693.

[11] Kwon, T., et al., Design and implementation of a simulator based on a cross-layer protocol between MAC and PHY layers in a WiBro Compatible. IEEE 802.16 e OFDMA system. Communications Magazine, IEEE, 2005.

[12]. Al-Khusaibi, H., Al-Wardi, F., Sawalha, F., \& Xiang, W. (2006, May). Experiment and Analysis on the Comparison of the IEEE 802.11 a and $802.11 \mathrm{~g}$ Wireless Local Area Networks. In 2006 IEEE International Conference on Electro/Information Technology (pp. 1316). IEEE.

[13]. Pamungkas, W., \& Suryani, T. (2018, March). Doppler effect in VANET technology on high user's mobility. In 2018 International Conference on Information and Communications Technology (ICOIACT) (pp. 899-904). IEEE.

. [14] Qureshi, S. U. (1985). Adaptive equalization. Proceedings of the IEEE, 73(9), 1349-1387.

\section{Appendix 1}

$\%$ This program simulate the ieee $802.11 \mathrm{p}$ for velocity range $[25,400 \mathrm{~km} / \mathrm{h}]$

$\%$ clear all;

carrier_num $=64$;

ts $=0.00000000008$;

$\mathrm{V}=1$;

for $\mathrm{fd}=136: 136: 2183$

FFT_size=64; \% IFFT/FFT

carrier $=[-26:-11: 26]$

carrier $=[2: 2739: 64]$

pilot $=[-21:-7: 7: 21]$

pilot $=[8,22,44,58]$

pilot_num=length(pilot); \% pilotlength

data $=[-26:-22$-20:-8 -6:-1 1:6 8:20 22:26];

data $=[2: 7$ 9:21 23:27 39:43 45:57 59:64];

data_num=length(data); \% data length

symbol num $=1000 ; \%$ OFDM symbol

frame_num $=500 ; \%$

CP_size $=16$; \% prefix cyclic

$\mathrm{T}=8 \mathrm{e}-6 ; \% \mathrm{OFDM}$

$\mathrm{Tu}=6.4 \mathrm{e}-6 ; \%$

$\mathrm{Tg}=1.6 \mathrm{e}-6 ; \%$

$\mathrm{SNR}=0: 2: 80 ; \%$ snr range

N_num=data_num*symbol_num;

$\%$ BER1=[];

BER2=[];

BitsTx=round(rand $\left(1, N \_n u m\right)$ );

Tx_BPSK=BitsTx.*2-1;

Tx_SP=reshape(Tx_BPSK,symbol_num,data_num);

pilot_Bits=round(rand(1,symbol_num) $)$;

pilot_symbol=(pilot_Bits.*2-1).';

$\%$

pilot_ofdm=zeros(symbol_num,FFT_size);

pilot_ofdm(:,pilot)=repmat(pilot_symbol,1,pilot_num);

pilot_ofdm $(:$,data $)=T x \_S P$;

training_symbol_num $=2$;

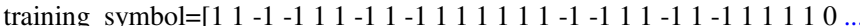

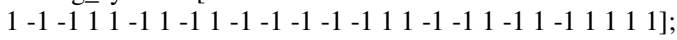

training_ofdm $1=$ zeros $(1$, carrier_num);

training_ofdm $1(:, 1: 53)=$ training_symbol

training_ofdm $=$ zeros $(1$, carrier_num)

training ofdm $(:, 1: 38)=$ training ofdm1 $(:, 27: 64)$;

training_ofdm $(:, 39: 64)=$ training_ofdm $1(:, 1: 26)$;

training symbols $=$ cat $(1$, training ofdm,training ofdm);

training_Txdata=cat $(1$, training_symbols,pilot_ofdm $) ; \% 12 * 64 ? ? ? ?$
IFFT_ofdm=ifft(training_Txdata,FFT_size,2);

CP_ofdm=zeros(symbol_num+training_symbol_num,carrier_num+CP_size); $\mathrm{CP}$ ofdm $(:, 1: \mathrm{CP}$ size $)=\mathrm{IFFT}$ ofdm $(:, \mathrm{FFT}$ size-CP size+1:FFT_size $)$;

CP_ofdm $\left(:, C P \_s i z e+1:\right.$ carrier_num+CP_size $)=I F F T \_o f d m$;

Tx_ofdm $=$ reshape $\left(C P \_o f d m, 1,(\right.$ carrier_num+CP_size $) *($ symbol_num+training symbol_num));

Ch=rayleighchan(fd,ts);

Tx_chan=filter(Ch,Tx_ofdm);

$\mathrm{d} 1=4 ; \mathrm{a} 1=0.2 ; \mathrm{d} 2=\overline{5} ; \mathrm{a} 2=0.3 ; \mathrm{d} 3=6 ; \mathrm{a} 3=0.4 ;$

$\mathrm{d} 4=7 ; \mathrm{a} 4=0.5$;

copy $1=\operatorname{zeros}\left(\operatorname{size}\left(\mathrm{Tx} \_\right.\right.$ofdm $\left.)\right)$;

for $\mathrm{i}=1+\mathrm{d} 1$ :length(Tx_ofdm)

copy $1(\mathrm{i})=\mathrm{a} 1 * \mathrm{Tx} \_$ofdm $(\mathrm{i}-\mathrm{d} 1)$;

end

copy $2=\operatorname{zeros}\left(\operatorname{size}\left(\mathrm{Tx} \_\right.\right.$ofdm $\left.)\right)$

for $\mathrm{i}=1+\mathrm{d} 2$ :length(Tx_ofdm)

copy $2(\mathrm{i})=\mathrm{a} 2 * \mathrm{Tx} \_$ofdm $(\mathrm{i}-\mathrm{d} 2)$;

end

copy3 $=$ zeros $\left(\operatorname{size}\left(T x \_o f d m\right)\right)$;

for $\mathrm{i}=1+\mathrm{d} 3$ :length(Tx_ofdm)

copy3(i) = a3*Tx_ofdm $(i-d 3)$;

end

copy4 $=$ zeros $\left(\operatorname{size}\left(T x \_o f d m\right)\right)$;

for $\mathrm{i}=1+\mathrm{d} 4$ : length(Tx_ofdm)

copy4(i) $=$ a4 $*$ Tx_ofdm $(i-d 4)$;

end

Tx_data $=$ Tx_ofdm + copy $1+$ copy $2+$ copy $3+$ copy 4

$\%$

for $\mathrm{s}=1$ :length $(\mathrm{SNR})$

Tx_signal_power $=\operatorname{var}\left(\mathrm{Tx} \_\right.$data $)$;

linear SNR $=10^{\wedge}(\mathrm{SNR}(\mathrm{s}) / 10)$

noise_sigma $=$ Tx_signal_power/linear_SNR;

noise scale factor $=\operatorname{sqrt}\left(\right.$ noise $\_$sigma);

noise $=\operatorname{randn}\left(1, \text { length }\left(T x \_d a t a\right)\right)^{*}$ noise_scale_factor;

Rx_ofdm $=$ Tx_data + noise

Rx_ofdm=awgn $\left(T x \_c h a n, s\right.$, 'measured');

Rx_SP=reshape(Rx_ofdm,symbol_num+training_symbol_num,carrier_num+ CP_size);

$\%$

DeCP_ofdm=zeros(symbol_num+training_symbol_num,carrier_num); DeCP_ofdm $(:, 1:$ carrier_num $)=R x \_S P\left(:, C P \_s i z e+1:\right.$ carrier_num+CP_size $)$; $\%$

FFT_ofdm=fft(DeCP_ofdm,FFT_size,2)

Rx_training_symbols=FFT_ofdm $(1: 2$,data $)$

Rx data_carrier=FFT_ofdm(3:symbol_num+training_symbol_num,data);

Rx_data_carrier_PS=reshape(Rx_data_carrier,1,N_num);

$\%$--------BPSK

demod_out2=zeros $(1$, length $(\mathrm{Rx}$ _data_carrier_PS $)$ )

for $\mathrm{q}=1$ : length(Rx_data_carrier_PS)

if abs(Rx_data_carrier_PS(q)-1)-abs(Rx_data_carrier_PS(q)+1)<=0

demod_out $2(\mathrm{q})=1$

else

demod_out $2(q)=0$;

end

$\%$ ber_count $1=0$;

ber_count $2=0$;

for $\mathrm{w}=1: \mathrm{N} \_$num

if $\operatorname{BitsTx}(\mathrm{w}) \sim=$ demod_out $2(\mathrm{w})$

ber_count $2=$ ber count $2+1$;

end

end

BER2=[BER2,ber_count2/N_num];

end

hold on

if BER2<0.8

$\mathrm{V}=\mathrm{fd} * 0.1838$;

$\operatorname{display}(\mathrm{V})$;

legend('Channel BER',3*fd/59);

hold on

semilogy(SNR,BER2,'-

r','linewidth',2,'markerfacecolor','g','markersize',8,'markeredgecolor','g');grid; $\%$ semilogy(SNR,BER1,'-og',SNR,BER2,'-*r');

end

grid on;

title('BER vs SNR'); 
xlabel('SNR/dB');

ylabel('BER');

legend(['BER, ','BPSK,',-','V=',num2str(V),'km/h']);

hold off

end

grid on;

title('BER vs SNR');

xlabel('SNR/dB');

ylabel('BER');

legend(['BER, N = ',symbol_num, ' ','BPSK','-',3.6*3*fd/59]);

legend('Channel BER');

\section{appendix 2}

$\%$ program to simulate the iee 802.2 ofdm

clear all; close all; clc;

$\% \%$ OFDM simulation

$\%$ By. MOUNTACIRI ABDERRAHIMr

$\%$ Initializing parameters

Nsc = input('OFDM symbol size (Number of subcarriers) $\mathrm{N}=$ ');

$\%$ OFDM symbol size (Number of subcarriers).

$\mathrm{M}=$ input('Modulation order $\mathrm{M}=$ '); \% Modulation order

$\mathrm{Nsmb}=$ input('Number of OFDM symbols to be simulated = ');

$\%$ Number of OFDM symbols to be simulated

$\mathrm{Ne}=10000$

; \% Number of bits in error

str $=0$;

stp=input('SNR starts at $0 \mathrm{~dB}$, with step size $[\mathrm{dB}]=$ =');

Esnr=input('Last value of SNR [dB] =');

$\% \%$ Modems Generation

$\mathrm{ht}=\operatorname{modem} . q \operatorname{mmod}(\mathrm{M}) ; \%$ Transmitter modem

$\mathrm{hr}=\operatorname{modem} . q a m d e m o d(\mathrm{M}) ; \%$ Receiver modem

$\operatorname{disp}\left({ }^{\prime} \% \% \% \% \% \% \% \% \% \% \% \% \% \% \% \% \% \% \% \% \% \% \% \% \% \% \% \% \% \% \% \%\right.$ $\% \% \% \% \% \% \% \% \% \% \% \% \% \%$

$\operatorname{disp}\left({ }^{\prime} \% \% \% \% \% \% \% \% \% \% \% \% \% \% \% \% \% \% \% \% \% \% \% \% \% \% \% \% \% \% \% \% \%\right.$ $\% \% \% \% \% \% \% \% \% \% \% \% \% \%$ );

disp( $\% \% \% \% \% \% \% \% \% \% \% \% \% \% \% \% \% \% \% \% \% \% \% \% \% \% \% \% \% \% \% \% \%$

$\% \% \% \% \% \% \% \% \% \% \% \% \% \%$ );

disp('OFDM Simulation using Monte Carlo for the BER evaluation');

disp(['Modulation family = ',num2str(M),'-',ht.type]);

disp(['Number of Subcarriers N = ',num2str(Nsc)]);

$\operatorname{disp}([$ 'SNR = ',num2str(str), ':',num2str(stp), ':',num2str(Esnr)])

disp $(1 \% \% \% \% \% \% \% \% \% \% \% \% \% \% \% \% \% \% \% \% \% \% \% \% \% \% \% \% \%$ $\% \% \% \% \% \% \% \% \% \% \% \% \% \%$;);

$\operatorname{disp}\left({ }^{\prime} \% \% \% \% \% \% \% \% \% \% \% \% \% \% \% \% \% \% \% \% \% \% \% \% \% \% \% \% \% \% \% \% \%\right.$ $\% \% \% \% \% \% \% \% \% \% \% \% \% \%$

disp( $\% \% \% \% \% \% \% \% \% \% \% \% \% \% \% \% \% \% \% \% \% \% \% \% \% \% \% \% \% \% \% \%$

$\% \% \% \% \% \% \% \% \% \% \% \% \%$ );

$\mathrm{c}=0$;

for snr=str:stp:Esnr

$\mathrm{c}=\mathrm{c}+1$;

$\% \%$ Monte Carlo Simulation loop

disp(['STEP ',num2str(c),' of ',num2str(length(str:stp:Esnr)),' :Processing SNR $=$ ',num2str(snr)]);

$\mathrm{nEr}=0 ; \%$ Number of collected errors

$\mathrm{nSmb}=0 ; \%$ Number of simulated OFDM symbols

while $((\mathrm{nEr}<\mathrm{Ne}) \& \&(\mathrm{nSmb}<\mathrm{Nsmb}))$

$\%$ Transmitter

Dg=randi([0 M-1],1,Nsc); \% Data Generation

Dmod= modulate $(\mathrm{ht}, \mathrm{Dg}) ; \%$ Baseband modulation (mapping)

dAM mod=ifft(Dmod,Nsc);

$\%$ Adding Noise using AWGN

dAM_mod_noisy=awgn(dAM_mod,snr,'measured');

$\%$ Amplitude demodulation (DFT using fast version FFT)

amdemod=fft(dAM_mod_noisy,Nsc);

$\mathrm{y}=$ demodulate(hr,amdemod);

$[\mathrm{n}, \mathrm{r}]=$ biterr$(\mathrm{Dg}, \mathrm{y})$;

$\mathrm{nEr}=\mathrm{nEr}+\mathrm{r}$;

$\mathrm{nSmb}=\mathrm{nSmb}+1$;

end

berRsit $(\mathrm{c})=\mathrm{nEr} /(\log 2(\mathrm{M}) * \mathrm{nSmb})$

end

scatterplot(Dmod); title('constellation for transmitted data ');

figure(1)

$\%$ scatterplot(Dmod);

figure(2)

[rows_ifft_data cols_ifft_data]=size(dAM_mod);

len_ofdm_data $=$ rows_ifft_data*cols_ifft_data

$\%$ Signal OFDM réel à transmettre

ofdm_signal $=$ reshape $\left(d A M \_m o d, 1\right.$, len_ofdm_data $)$;

plot(real(ofdm_signal)); xlabel('Time'); ylabel('Amplitude');

snr=str:stp:Esnr; figure(3)

semilogy(snr,berRslt,'-

g','linewidth',2,'markerfacecolor','g','markersize',8,'markeredgecolor','g'); grid; title('OFDM Bit Error Rate vs SNR');

ylabel('Bit Error Rate');

xlabel('SNR [dB]');

legend(['BER, $\mathrm{N}=$ ', num2str(Nsc),' ',num2str(M),'-',ht.type]);

n_bits=Ne;

$\mathrm{EbNodB}=40$;

$\mathrm{k}=\log 2(\mathrm{M})$;

$\mathrm{EbNo}=10^{\wedge}(\mathrm{EbNodB} / 10)$

$\mathrm{y}=$ Dmod;

$\%$ Channel

$\left.\mathrm{Eb}=\mathrm{mean}((\operatorname{abs}(\mathrm{y})))^{\wedge} 2\right) / \mathrm{k}$;

sigma $=\operatorname{sqrt}(\mathrm{Eb} /(2 * \mathrm{EbNo}))$

$\mathrm{w}=\operatorname{sigma} *(\operatorname{randn}(\mathrm{Nsc}, 1)+1 \mathrm{i} * \operatorname{randn}(\mathrm{Nsc}, 1))$

$\mathrm{h}=(1 / \mathrm{sqrt}(2)) *\left(\operatorname{randn}(\mathrm{Nsc}, 1)+1 \mathrm{i}^{*} \operatorname{randn}(\mathrm{Nsc}, 1)\right) ; \mathrm{h} 1=\operatorname{transpose}(\mathrm{h})$;

$\mathrm{r}=\mathrm{h} 1$. $^{*} \mathrm{y}+\operatorname{transpose}(\mathrm{w})$

$\%$ scatterplot(r)

$\%$ Receiver

$\mathrm{r}=\operatorname{transpose}(\mathrm{r}) . / \mathrm{h}$

figure(4)

scatterplot(r);title('constellation for receiver data with rayleigh channel'); snr=str:stp:Esnr;

figure(4)

semilogy(snr,berRslt,'-

g','linewidth',2,'markerfacecolor','g','markersize',8,'markeredgecolor','g');grid; title('OFDM Bit Error Rate vs SNR');

ylabel('Bit Error Rate');

xlabel('SNR [dB]');

legend(['BER, $N$ = ', num2str(Nsc),' ',num2str(M),'-',ht.type]);

appendix 3

$\div \%$ 등 $\%$ hannel mode

close all; clc; clear; $\mathrm{NO}=20$

№mber of scatters

$\mathrm{NFFT}=2048 ;$

은-th scatter(40??) generator

speed $=2 ; \% \mathrm{Km} / \mathrm{h}$

num_path $=40$;

$\mathrm{NFFT}=4 * 256$;

Nsamples $=\mathrm{NFFT} ; \% 4096$

Tslot $=16 e-6 ; \% 1 e-2 / 15$

Tchip = Tslot $/ 1500$

: Tchip $=333 e-6$;

Tslot $=$ Tchip $* 256 ;$

sdat $=[1:$ Nsamples $]$;

fs $=1 /$ Tslot;

tdat $=$ sdat $/ \mathrm{fs}$;

$\mathrm{fd}=($ speed $/ 3.6) * 5.9 \mathrm{e} 9 / 3 \mathrm{e} 8$;

const_seed $=$ randn (' seed');

tau $=[0]$;

flat seq $=$

flat fadingl (1, N0, fd, tdat, tau, 1, const seed) ;

ospeed 40

figure

subplot $(3,1,1)$

plot (tdat, (abs (flat_seq)))

grid on;

xlabel ('Time (sec) ')

ylabel ('Amplitude ')

title('Velocity $=2 \mathrm{~km} / \mathrm{h}$ ')

ospeed 80

speed $=180$

$\mathrm{fd}=($ speed $/ 3.6) * 2.45 \mathrm{e} 9 / 3 \mathrm{e} 8$

flat seq $=$

flat fadingl ( $1, \mathrm{~N} 0, \mathrm{fd}$, tdat, tau, 0, const_seed) ;

subplot $(3,1,2)$

plot (tdat, (abs (flat_seq)));

grid on;

xlabel ('Time (sec) ')

ylabel ('Amplitude ')

title('Velocity $=120 \mathrm{~km} / \mathrm{h}$ ')

ospeed 200

speed $=400 ;$

$\mathrm{fd}=($ speed $/ 3.6) * 5.9 e 9 / 3 e 8$ 
flat seq $=$

flat_fading1 (1,N0,fd, tdat, tau, 1, const_seed) ; subplot $(3,1,3)$

plot (tdat, (abs (flat_seq)));

grid on;

xlabel ('Time (sec)')

ylabel ('Amplitude ')

title ('Velocity $=400 \mathrm{~km} / \mathrm{h}$ ')

[pwr freq] $=\operatorname{psd}(f l a t \operatorname{seq}(1,:), \mathrm{NFFT}, \mathrm{fs})$;

flat_spec $=$ abs $($ fftshift $(\operatorname{pwr}(:, 1)))$;

$\operatorname{xdat} \overline{1}=($ freq-freq $($ length $($ freq $) / 2+1)) /$ fd;

xdat $2=\left[\begin{array}{ll}-1 & 1\end{array}\right] ;$

ideal curve $2=\left[\begin{array}{ll}1 & 1\end{array}\right] /(2 * f d)$

figurēe();

plot (xdat1, $10 * \log 10$ (flat_spec), '-x');

hold on;

plot (xdat2, ideal_curve2, 'r-', 'linewidth', 2);

xlabel ('Frequency normalized by max. Doppler shift')

ylabel ('amplitude (dB) ')

set (gca, 'ylim', [-120 40])

set (gca,' 'xlim', [ $\left.\begin{array}{ll}-2 & 2\end{array}\right]$ )

grid on

legend('Flat fading', 'Ideal', 3)

$\left[\right.$ pwr freq] $=\operatorname{psd}\left(f l a t \_s e q(1,:), N F F, f s\right)$;

flat $\operatorname{spec}=$ abs (fftshift $(\operatorname{pwr}(:, 1)))$;

$\operatorname{xdat} \overline{1}=($ freq-freq $($ length $($ freq $) / 2+1)) / f d$;

xdat2 $=\left[\begin{array}{ll}-1 & 1\end{array}\right] ;$

ideal_curve $2=\left[\begin{array}{ll}1 & 1\end{array}\right] /(2 * \mathrm{fd})$;

figure ();

plot (xdat1, $10 * \log 10$ (flat spec), '-x');

hold on;

plot(xdat2, ideal curve2, 'r-', 'linewidth', 2);

xlabel ('Frequency normalized by max. Doppler shift')

ylabel ('amplitude (dB)')

set (gca, 'ylim', [-120 40])

set (gca, 'xlim', [-2 2])

grid on

legend('Flat fading', 'Ideal', 3)

음akes model

$t$ state $=0$;

speed $=40$;

$\mathrm{fd}=($ speed $/ 3.6) * 5.9 \mathrm{e} 9 / 3 \mathrm{e} 8$

[jakes_seq,t_state]=Jakes_Flat(fd, Tslot, Nsamples, t_state $, 1,0) \overline{\text {; }}$

subplot (311), plot ([1:Nsamples]*Tslot,

$10 * \log 10($ abs (jakes seq) $))$

title(['Jakes Model, f d=', num2str(fd), 'Hz, T s', num2str(Tslot), 's']);

axis([0 0.05 -20 10]), xlabel('time(s)'),

ylabel ('amplitude')

figure ();

subplot $(3,1,1)$

plot (tdat,

$\left.10 * \log 10\left(\operatorname{abs}\left(j a k e s \_s e q / \max \left(\operatorname{abs}\left(j a k e s \_s e q\right)\right)\right)\right)\right)$;

grid on;

xlabel ('Time (sec)')

ylabel ('Amplitude ')

title ('Velocity $=40 \mathrm{~km} / \mathrm{h}$ ')

axis $\left(\left[\begin{array}{llll}0 & 0.09 & -30 & 10\end{array}\right]\right)$;

ospeed 80

speed $=120$

$\mathrm{fd}=($ speed $/ 3.6) * 5.9 \mathrm{e} 9 / 3 \mathrm{e} 8$;

flat seq $=$

flat fadingl (1,N0,fd, tdat, tau, 0, const_seed) ;

subp $\bar{l}$ ot $(3,1,2)$

plot (tdat,

$10 * \log 10\left(\right.$ abs $\left(\right.$ flat_seq) $\left.\left./ \max \left(\operatorname{abs}\left(j a k e s \_s e q\right)\right)\right)\right)$;

grid on;

xlabel ('Time (sec) ')

ylabel ('Amplitude ')

title ('Velocity $=120 \mathrm{~km} / \mathrm{h}$ ')

axis ([0 $0.09-30$ 10]);

\%speed 200

speed $=400 ;$

$\mathrm{fd}=(\mathrm{speed} / 3.6) * 2.45 \mathrm{e} 9 / 3 \mathrm{e} 8$ flat seq $=$

flat_fadingl (1,N0,fd, tdat, tau, 1, const_seed); subp $\bar{l}$ ot $(3,1,3)$

plot (tdat

$10 * \log 10\left(\right.$ abs $\left(\right.$ flat_seq) $\left.\left./ \max \left(\operatorname{abs}\left(j a k e s \_s e q\right)\right)\right)\right)$;

grid on;

xlabel ('Time (sec) ')

ylabel ('Amplitude ')

title ('Velocity $=400 \mathrm{~km} / \mathrm{h}$ ')

$\operatorname{axis}\left(\left[\begin{array}{llll}0 & 0.09 & -30 & 10\end{array}\right]\right)$;

$[\mathrm{pwr}$ freq] $=\operatorname{psd}(\mathrm{flat} \operatorname{seq}(1,:), \mathrm{NFFT}, \mathrm{fs})$;

flat spec $=$ abs (fftshift $(\operatorname{pwr}(:, 1)))$;

$\operatorname{xdat} \overline{1}=($ freq-freq $($ length $($ freq $) / 2+1)) / f d$;

$x$ dat $2=\left[\begin{array}{ll}-1 & 1\end{array}\right]$;

ideal_curve2 $=\left[\begin{array}{ll}1 & 1\end{array}\right] /(2 * f d)$;

figurēe ();

plot (xdat1, $10 * \log 10$ (flat spec), '-x');

hold on;

plot (xdat2, ideal curve2, 'r-', 'linewidth', 2);

xlabel ('Frequency normalized by max. Doppler shift')

ylabel ('amplitude ')

set (gca,'ylim', [-120 40])

set (gca,' 'xlim', [-2 2])

grid on

legend('Flat fading', 'Ideal', 3)

$[$ pwr freq] $=\operatorname{psd}(\mathrm{flat}$ _seq $(1,:), \mathrm{NFFT}, \mathrm{fs})$;

flat spec $=$ abs (fftshift $(\operatorname{pwr}(:, 1)))$;

$\operatorname{xdat} \overline{1}=($ freq-freq $($ length $($ freq $) / 2+1)) / f d$;

xdat2 $=\left[\begin{array}{ll}-1 & 1\end{array}\right]$;

ideal curve $2=\left[\begin{array}{ll}1 & 1\end{array}\right] /(2 * f d)$.

figurēe ();

plot (xdat1, $10 * \log 10$ (flat spec), '-x');

hold on:

plot (xdat2, ideal_curve2, 'r-', 'linewidth', 2);

xlabel ('Frequency normalized by max. Doppler shift')

ylabel ('amplitude ')

set (gca, 'ylim', [-120 40])

set (gca, 'xlim', [-2 2])

grid on

legend('Flat fading', 'Ideal', 3)

[pwr freq] $=\operatorname{psd}(j$ akes $\operatorname{seq}(1,:), \operatorname{NFFT}, \mathrm{fs})$;

jakes_spec $=$ abs $($ fftshift $(\operatorname{pwr}(:, 1)))$;

xdat $1=($ freq-freq $($ length $($ freq $) / 2+1)) / f d$;

tmp $=1-1 e-10$;

xdat2 = linspace (-tmp, tmp, 100);

ideal curve1 = $-10 * \log 10((2 *$ pi*sqrt $(1-$

$\left.\left.\operatorname{abs}(x \bar{d} a t 2) \cdot{ }^{\wedge} 2\right)\right)$ ) ;

figure ();

plot (xdat1, $\left.10 * \log 10\left(j a k e s \_s p e c\right), \quad '-x^{\prime}\right)$;

hold on;

plot (xdat2, ideal_curve1, 'r-', 'linewidth', 2);

xlabel ('Frequency normalized by max. Doppler shift')

ylabel ('Amplitude ')

set (gca,'ylim', [-120 40])

set (gca, 'xlim', [-2 2])

grid on

legend('Jakes fading',' Ideal', 3)

appendix 4

lms algorithm

$\mathrm{N}=6000$;

information sequence

$\mathrm{K}=11$;

11 tap coefficients

1 channel

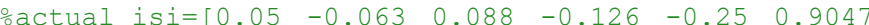

$0.250 \overline{0} .126 \quad 0.038$

0.088]; 응 channel response has

oChannel complex

\%actual_isi $=[0.0410+0.0109 j$

$0.7920+0.1281 j$

$0.3960+0.0871 j \quad 0.2715+0.0498 j \quad 0.2291+0.0414 j$

$0.1287+0.0154 j 0.1032+0.0119 j] ;$

actual isi $=\left[\begin{array}{lllllll}0.005 & 0.009 & -0.024 & 0.854 & -0.218 & 0.049\end{array}\right.$ -

$0.016]$

Num_of_realizations $=70 ; \quad \frac{\circ}{0}$ Learning times

mse $\operatorname{av}=\operatorname{zeros}(1, \mathrm{~N}-\mathrm{K}+1)$;

$\sin =30$;

delta $k=0.000001$;

sigpower=10;

$\mathrm{d}=1$;

distance of
oAM is a European space for a 


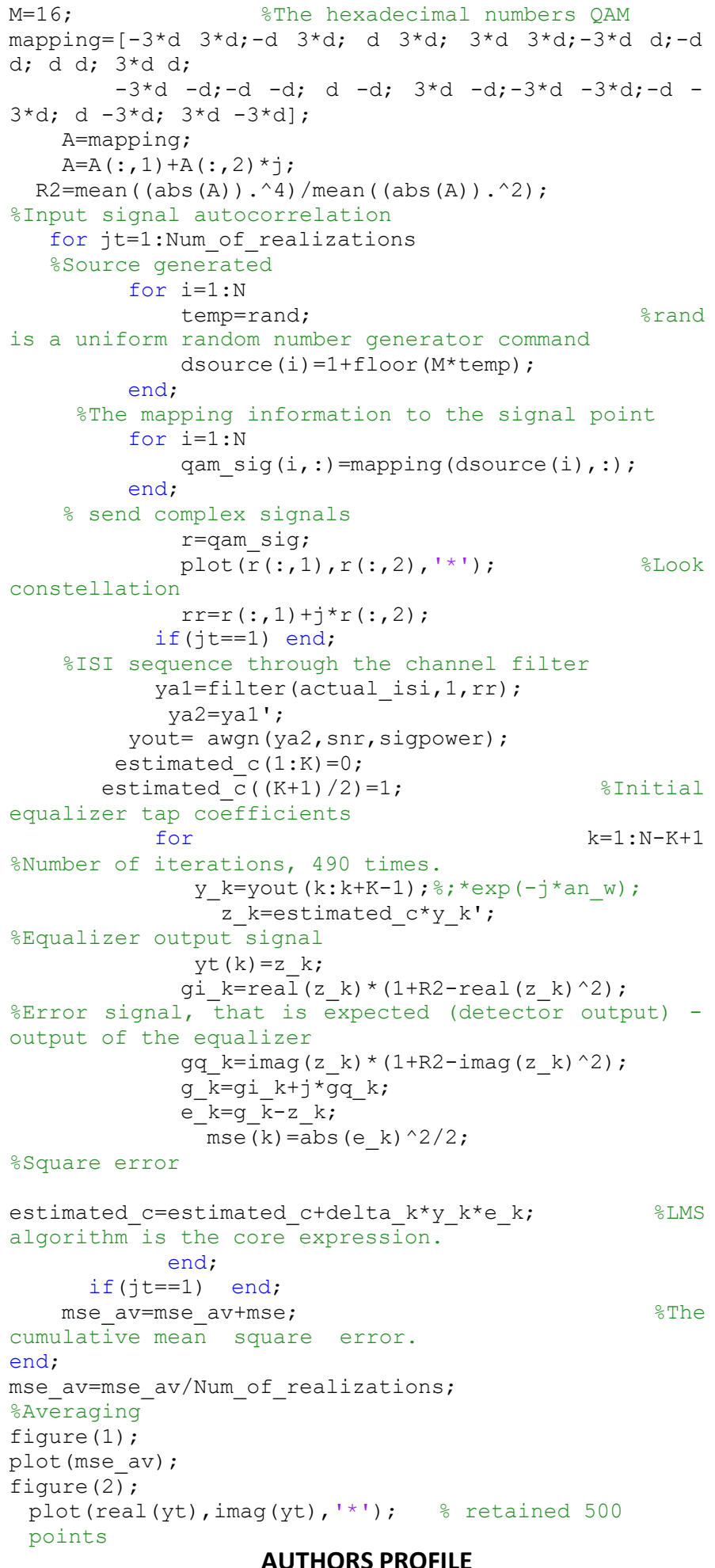

\section{AUTHORS PROFILE}

Mountaciri abderrahim is Professor of electronics leaving the ENSET of MOHAMMEDIA in 1993, associate professor in electrical engineering in 2005 of the ENSET RABAT practicing since 2007 in the preparatory class of the large engineering schools in settat moroc, these recharges are done in energy andtelecom network

El Mostafa MAKROUM is Professor in the faculty of the sciences and technology of Settat, Morocco. He received his M.S. degree in 2007 from the Mohammadia School of Engineering, University Mohammed V, Rabat, Morocco. He received his Ph.D. degree in Computers and Telecommunications from the Higher

National School of Electricity and Mechanics, University

Hassan II, Casablanca, Morocco. His current research concerns RFID antennas, propagation and EMC problems

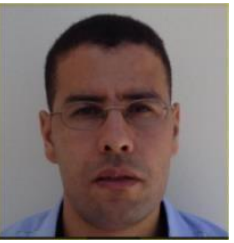

My Abdelkader Youssefi received his engineering degree in telecommunications from $\mathrm{Na}$ tional Institute of Telecommu nications (INPT), Rabat, Mo rocco, in 2003, and received his Ph.D. degree from Mohamma dia School of Engineers (EMI) at Mohammed V University in

2015. Between September 2003 and May 2016, he worked as a telecommunications engineer.

Currently, Youssefi is a teacher at Hassan I University, Settat, Morocco. His re search interests include cognitive radio, channel estimation, massive MIMO, OFDM and Internet of Things security in wireless networks.

Papa0011@@
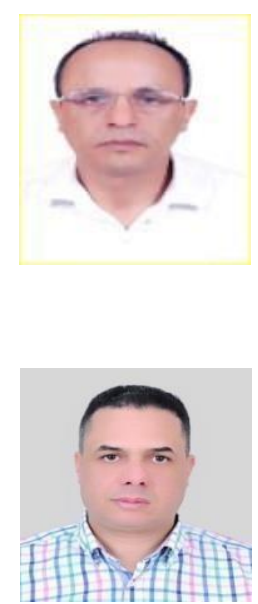
ISBN: 978-988-19253-7-4 